Case Report

\title{
Could Hallucinogens Induce Permanent Pupillary Changes in (Ab)users? A Case Report from New Zealand
}

\author{
Ahmed Al-Imam ${ }^{1,2}$ \\ ${ }^{1}$ Novel Psychoactive Substances Unit, Doctoral College, Hertfordshire University, Hertfordshire, UK \\ ${ }^{2}$ College of Medicine, University of Baghdad, Baghdad, Iraq \\ Correspondence should be addressed to Ahmed Al-Imam; tesla1452@gmail.com \\ Received 18 May 2017; Revised 18 July 2017; Accepted 19 July 2017; Published 17 August 2017 \\ Academic Editor: Norman S. Litofsky \\ Copyright (C) 2017 Ahmed Al-Imam. This is an open access article distributed under the Creative Commons Attribution License, \\ which permits unrestricted use, distribution, and reproduction in any medium, provided the original work is properly cited. \\ An eighteen-year-old female patient of the Caucasian ethnicity from Australasia presented with a persistently dilated pupil causing \\ her discomfort and occasional burning sensation when she is outdoors due to oversensitivity to sunlight. However, her pupillary \\ reaction to light (pupillary light reflex) was intact. The patient is a known user of psychedelic substances (entheogens) including \\ LSD, NBOMe, psilocybin, and DMT. The condition affects both eyes to the same extent. Thorough medical, neurological, and \\ radiological examinations, including an EEG and an MRI of the head and neck region, were completely normal. All these tests \\ failed to detect any pathophysiological or anatomical abnormalities. The patient is a known case of chronic endogenous depression \\ in association with attention deficit hyperactivity disorder, for which she is taking citalopram and Ritalin, respectively. There was \\ neither a family history nor a similar congenital condition in her family.
}

\section{Introduction}

Novel psychoactive substances (NPS), also known as research chemicals or designer drugs, are a group of substances, including chemicals that can either stimulate (stimulants) or inhibit (depressants) the nervous system, particularly the central nervous system $[1,2]$. According to the classification scheme reported by the European Monitoring Centre for Drugs and Drug Addiction (EMCDDA), NPS can be categorised into cannabis and cannabimimetic, phenethylamines, cathinones, tryptamines, piperazine, and pipradrol derivatives and a 7th miscellaneous group which is composed predominantly of CNS stimulants $[2,3]$. This taxonomy is based on the structural chemistry of 252 substances that were reported to the EMCDDA in between 1997 and 2012. The exponential rise in of the NPS phenomenon is considered to be correlated with the logarithmic growth of information and communication technology $[4,5]$.

The majority of these substances possess addictive properties. Hence, substance users and misusers may develop dependence syndrome, withdrawal manifestations, or adverse reactions [6]. These substances act on various neurotransmitters within the nervous system, including the central nervous system (CNS) and the peripheral nervous system (PNS) $[2,7]$. The key neurotransmitters are monoamines including dopamine, serotonin, and catecholamine. In fact, NPS exert their effects via their highly selective affinity towards monoamine transporters (MATs); MATs include serotonin transporter (SERT), dopamine transporter (DAT), and norepinephrine (NET) $[8,9]$.

MATs are located just around the synaptic cleft (perisynaptically); they are responsible for the reuptake of monoamines back from the synaptic cleft into the cytoplasm of the presynaptic neurons $[10,11]$. Therefore, the action of NPS on the body systems, both centrally and peripherally, can be attributed to changes achieved via MATs. NPS can induce several physiologic changes including ocular alterations [12], for example, morphometric variations in the dimension (diameter) of the pupillary aperture leading to either pupillary constriction (miosis) or dilation (mydriasis). 


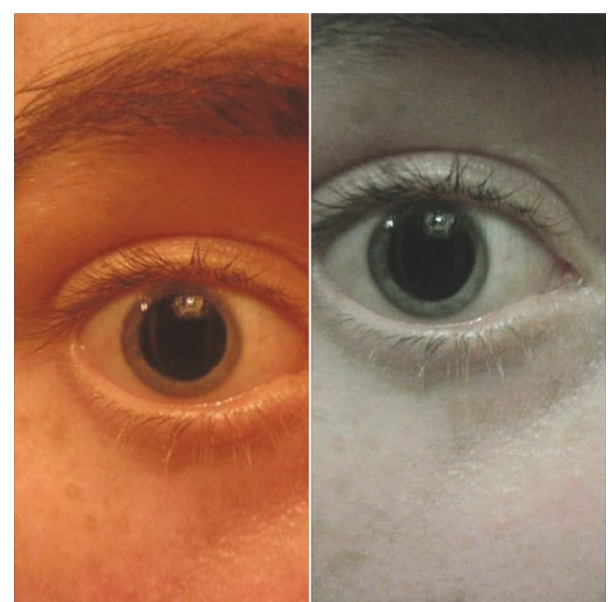

FIGURE 1: Dilated right pupil of a female psychedelics' user.

\section{Case Report}

The patient is an 18-year-old female of the Caucasian ethnicity; she is from Australasia, specifically New Zealand; she has light coloured skin of Fitzpatrick type-1 category $[13,14]$. She is a right-handed artist and of a potentially lefthemispheric cerebral dominance. She has a past history of substance use and misuse starting at the age of eleven years, smoking cannabis and hashish; at that age she developed an abnormally and continuously dilated pupil (Figure 1) leaving a thin rim of blue iridial tissue; the condition is affecting both eyes (bilaterally) though both pupils still react to light, including sunlight (i.e., pupillary light reflex is intact).

She had no complaints except for intolerance to sunlight, both direct and indirect, which mandates wearing $U V$ sunglasses for optimal protection of the retina as advised by a specialist optometrist. Hence, the patient is almost unbothered by her condition; she described it by saying " $m y$ eyes always look like this, I have constantly dilated pupils, haha, not even tripping." In fact, she considers her overall eye appearance as sexier than the normal eye. However, she was bothered of the potential eye and retinal damage from overexposure to sunlight.

The patient has correlated her eye condition with the use of hallucinogens, primarily with LSD (acid) and psilocybin mushrooms. Furthermore, her condition started at the age of eleven years in association with substance use. Hence, it is not congenital. In 1992, the 1st case of a bilateral congenital mydriasis was reported in the literature [15]. The patient has a past medical history, being treated with citalopram and Ritalin for the management of her chronic endogenous depression and attention deficit hyperactivity disorder (ADHD); she has been on these medications for several years. She also admitted using tramadol, opium, and opioid derivatives; these substances induce a paradoxical effect on her pupil leading to pupillary constriction. The patient had no prior history of head trauma, brain tumors, or any other neurological conditions. Furthermore, a thorough neurological and radiological exam was done; an MRI of the head and neck region was also entirely normal.

\section{Discussion}

In Renaissance Italy, Italian ladies used to apply a purified extract from the berries of Atropa belladonna as eye drops to both eyes; the purpose was to create artificially dilated pupils; it was considered a sign of beauty; belladonna is Italian for a beautiful lady [16, 17]. The extract contains anticholinergic substances including atropine, scopolamine, and hyoscyamine $[16,17]$. In this case presentation, the pupillary changes (mydriasis) were brought by the pathophysiologic effect of possibly more than one substance, primarily hallucinogenic agents that own sympathomimetic or parasympathomimetic-related properties [18]. Though it may still be considered as a sign of beauty, it increases the sensitivity towards sunlight (photosensitivity) particularly in fair skin individuals and Caucasians of Fitzpatrick skin type-1 and type-2 [19].

Changes in the pupillary dimensions are brought up by either an increment or a decrement in its diameter via the action of iridial smooth muscles, both longitudinal (dilator pupillae) and circular (sphincter pupillae) [7]. The autonomic nervous system (ANS), an integral component of the PNS, is responsible for the automatic (visceral) control of the pupillary aperture [20]. The sympathetic nervous system (SNS) usually mediates pupillary dilation (mydriasis), while the parasympathetic nervous system, via the oculomotor nerve (cranial nerve III) and its modulatory effect over the ciliary autonomic ganglion, mediates pupillary constriction (miosis) [20, 21]. Changes in the sympathetic or parasympathetic tone (neuronal activity) are brought up by changes via reflex mechanism (as in pupillary light reflex), emotional changes affecting the limbic system and the diencephalon specifically the hypothalamus, and the modulation of the neuronal activity in the midbrain specifically pretectal region and the Edinger-Westphal nucleus (accessory oculomotor nucleus). The last nucleus houses the presynaptic (preganglionic) parasympathetic motor neurons of the oculomotor nerve which innervate the sphincter pupillae iridial muscles $[7,22]$. Hence, this pathway, pretectal-accessory oculomotor nucleus, is a critical constituent of the pupillary light reflex; the afferent and efferent nerves of this pathway are the optic nerve and the oculomotor nerve, respectively; the reflex is considered to be a four-neuronal reflex pathway [23]. Accordingly, this reflex neuronal pathway controls the momentary changes (pupillary unrest under ambient light) in the pupillary aperture with a high accuracy and an ultimate speed (milliseconds) in response to variations in the illumination level of the surrounding environment [24, 25].

Certain pathophysiological changes can influence the pupillary aperture; these include the pupillary light reflex mechanism, the tone of SNS or PNS including their autonomic ganglion, and pathologies of the midbrain around the regions of the cerebral aqueduct of Sylvius including the regions of tectum and tegmentum, hypothalamic region, limbic system, and higher centres. For instance, Horner's syndrome is a condition in which damage affects the function of the upper segments (cervicothoracic) of the paravertebral sympathetic chain, leading to dilated pupil on the ipsilateral side in addition to ipsilateral eyelid ptosis and hemifacial 
anhidrosis [26]. Several conditions may result in Horner's syndrome, including central (CNS) and peripheral (PNS and ANS), including syringomyelia, multiple sclerosis, brain tumors, encephalitis, lateral medullary syndrome, cervical rib, thyroid tumors and thyroidectomy, bronchogenic carcinoma, tube thoracostomy, carotid artery dissection, cavernous sinus thrombosis, middle ear infections, sympathectomy, and nerve block procedures $[26,27]$. All these pathological conditions operate either centrally at the level of the hypothalamospinal tract or at the presynaptic sympathetic neurons, or peripherally at the postsynaptic sympathetic neurons. Horner's syndrome can occur either unilaterally or bilaterally; several tests are used to diagnose this syndrome as in the cocaine drop test [28].

Several conditions and agents can cause mydriasis; these are injury to the eye and associated neural elements, anticholinergic medications and chemicals such as atropine and scopolamine, the elevated level of oxytocin hormone, and drug use and misuse $[15,29]$. Drugs include cocaine (crack), MDMA (ecstasy), hallucinogens, methamphetamine (crystal meth), and Toradol (ketorolac). Hallucinogenic drugs and entheogen are not limited to LSD (acid), NBOMe (nbomb), and dimethyltryptamine (DMT) [30]. Stimulants (as in cocaine) and hallucinogens act via increasing the levels of serotonin mainly by acting on SERT located centrally (CNS) [31]. In fact, these drugs that may lead to an overall increase in 5-hydroxytryptamine (serotonin) or a subsequent effect on $5-\mathrm{HT}_{2 \mathrm{~A}}$ receptor will exert a mydriatic effect, as in the case of psychedelics [11, 31]. Other conditions leading to abnormally dilated pupil include benign episodic unilateral mydriasis, cranial nerves neuropathy, traumatic brain injury, and mydriatic agents used for ophthalmologic examination such as tropicamide [32]. Oxytocin, the love hormone, can induce mild to moderate mydriatic effect; oxytocin is related to intimate emotional and social interactions. Hence, it increases in bursts during sexual intercourse; Pitocin (oxytocin) is also medicinally used to induce uterine contraction to either facilitate or induce a normal vaginal delivery $[33,34]$.

Similar cases were reported in drug fora, particularly from abusers of psychedelic substances including both males and females. One of the threads included this comment " $M y$ friend has one pupil increased in size and one decreased. Permanently... It happened to him after I made a cacao from weed and we tripped our balls. It looks funny" $[35,36]$. In fact, some psychedelic users reported that they were able to control the size of their pupillary apertures voluntarily; a male has commented "I can change the size of my pupils while looking in the mirror" $[35,36]$. This is remarkable given the fact that iridial muscles are strictly controlled involuntarily by autonomic innervation [36]. Perhaps, some neuronal (or neurochemical) modulation exists in psychedelic (ab)users. Contrary to psychedelic users, users and misusers of opium and opioid substances experience a constricted (miotic) pupil, or even a pinpoint pupillary aperture [37]. Heroin, fentanyl, codeine, methadone, and morphine act via stimulation of the PNS [38]. The New Zealander patient presented in this manuscript admitted taking these substances (opioids) too; she has also confirmed that her pupils could still react with some degree of constriction. She commented saying "I do take tramadol and codeine as well, but they do the opposite to the eyes."

Clinical examination, including a thorough neurological exam, failed to detect any abnormalities apart from the bilaterally dilated pupils. Furthermore, MRI of the regions of the craniocervical and thoracic region could not detect any pathology. It is likely that changes may exist at a cellular level that cannot be detected with the conventional methods, or at the centrally located nuclei in the midbrain and the hypothalamus. Functional MRI (fMRI) can be useful to detect these changes, but it was not available in the medical institute at which the patient was examined [39, 40]. Transcranial magnetic stimulation (TMS) might be of value in detecting lesions in the limbic system, temporal lobe, or the prefrontal cortex. However, TMS is not suitable to detect deep-seated lesions in the hypothalamus or the midbrain [41, 42].

This level-of-evidence of this manuscript is level-5 in accordance with the classification system imposed by the Oxford Centre for Evidence-Based Medicine (CEBM) [43, 44].

\section{Conclusion}

The case of the New Zealander female presented in this manuscript is one of the few cases documented in the body of literature. The patient had a frequent use of NPS, psychedelics, and other psychoactive chemicals including antidepressant medications. It can be inferred that the burden of (ab)use of hallucinogenic and other NPS chemicals is not to be underrated, particularly in the developed world, including Australasia. These substances can be abused as early as childhood leading to irreversible consequences including adverse pathophysiological changes of body systems, dependence syndrome, incidents of intoxications, fatalities, and sudden death. The magnitude of these hazards is obscure in relation to the developing countries, including the Middle East, Asia, Africa, and Latin America. In connection with the Middle East, more in-depth epidemiological investigations are mandatory to infer an estimate in relation to the spread of psychedelic (ab)use.

\section{Conflicts of Interest}

The author has no conflicts of interest to be declared.

\section{Acknowledgments}

Appreciation and gratitude are due to the administration of the Terence McKenna Page, a private group located on the Facebook social communication medium; the group is dedicated for psychedelics users. The author would also like to acknowledge efforts of Dr. Mayasa Mohammed Al-Hyali, for her insightful remarks in relation to Introduction of this manuscript.

\section{References}

[1] I. Brew, "Novel psychoactive substances," British Journal of General Practice, vol. 66, no. 644, p. 125, 2016. 
[2] P. Dargan and D. Wood, Eds., Novel Psychoactive Substances: Classification, Pharmacology and Toxicology, Academic Press, 2013.

[3] L. Orsolini, D. Papanti, R. Vecchiotti, A. Valchera, J. Corkery, and F. Schifano, "Novel psychoactive substances," European Psychiatry, vol. 33, pp. S59-S60, 2016.

[4] O. Corazza, F. Schifano, P. Simonato et al., "Phenomenon of new drugs on the internet: the case of ketamine derivative methoxetamine," Human Psychopharmacol: Clinical and Experimental, vol. 27, no. 2, pp. 145-149, 2012.

[5] O. Corazza, S. Assi, P. Simonato et al., "Promoting innovation and excellence to face the rapid diffusion of novel psychoactive substances in the EU: the outcomes of the ReDNet project," Human Psychopharmacology: Clinical and Experimental, vol. 28, no. 4, pp. 317-323, 2013.

[6] S. Fazel, P. Bains, and H. Doll, "Substance abuse and dependence in prisoners: A systematic review," Addiction, vol. 101, no. 2, pp. 181-191, 2006.

[7] M. D. Vaz, T. D. Raj, and K. D. Anura, Guyton \& Hall Textbook of Medical Physiology: A South Asian Edition, Elsevier Health Sciences, 2014.

[8] R. R. Gainetdinov and M. G. Caron, "Monoamine transporters: from genes to behavior," Annual Review of Pharmacology and Toxicology, vol. 43, no. 1, pp. 261-284, 2003.

[9] R. B. Rothman and M. H. Baumann, "Monoamine transporters and psychostimulant drugs," European Journal of Pharmacology, vol. 479, no. 1-3, pp. 23-40, 2003.

[10] N. V. Cozzi, A. Gopalakrishnan, L. L. Anderson et al., "Dimethyltryptamine and other hallucinogenic tryptamines exhibit substrate behavior at the serotonin uptake transporter and the vesicle monoamine transporter," Journal of Neural Transmission, vol. 116, no. 12, pp. 1591-1599, 2009.

[11] M. E. Liechti, "Novel psychoactive substances (designer drugs): overview and pharmacology of modulators of monoamine signalling," Swiss Medical Weekly, vol. 145, 2015.

[12] N. Hohmann, G. Mikus, and D. Czock, "Effects and risks associated with novel psychoactive substances," Deutsches Ärzteblatt International, vol. 111, no. 9, pp. 139-147, 2014.

[13] S. Eilers, D. Q. Bach, R. Gaber et al., "Accuracy of self-report in assessing Fitzpatrick skin phototypes I through VI," JAMA Dermatology, vol. 149, no. 11, pp. 1289-1294, 2013.

[14] S. Majewski, C. Carneiro, E. Ibler et al., "Digital dermoscopy to determine skin melanin index as an objective indicator of skin pigmentation," Journal of Surgical Dermatology, vol. 1, no. 1, 2016.

[15] P. Richardson and W. E. Schulenburg, "Bilateral congenital mydriasis," British Journal of Ophthalmology, vol. 75, no. 10, pp. 632-633, 1992.

[16] A. Abbaspour, P. Khadiv Parsi, F. Khalighi-Sigaroodi, and R. Ghaffarzadegan, "Optimization of atropine extraction process from atropa belladonna by modified bubble column extractor with ultrasonic bath," Iranian Journal of Chemistry and Chemical Engineering, vol. 35, no. 4, pp. 49-60, 2016.

[17] K. Dimitrov, D. Metcheva, and L. Boyadzhiev, "Integrated processes of extraction and liquid membrane isolation of atropine from Atropa belladonna roots," Separation and Purification Technology, vol. 46, no. 1-2, pp. 41-45, 2005.

[18] P. Śramska, A. Maciejka, A. Topolewska, P. Stepnowski, and $Ł$. P. Haliński, "Isolation of atropine and scopolamine from plant material using liquid-liquid extraction and EXtrelut ${ }^{\circledR}$ columns," Journal of Chromatography B, vol. 1043, pp. 202-208, 2017.
[19] M. Wilkes, C. Y. Wright, J. L. Du Plessis, and A. Reeder, "Fitzpatrick skin type, individual typology angle, and melanin index in an African population: steps toward universally applicable skin photosensitivity assessments," JAMA Dermatology, vol. 151, no. 8, pp. 902-903, 2015.

[20] K. T. Patton, Anatomy and physiology, Elsevier Health Sciences, 2015.

[21] A. Presland and J. Price, "Ocular anatomy and physiology relevant to anaesthesia," naesthesia \& Intensive Care Medicine, vol. 18, no. 1, pp. 27-32, 2017.

[22] C. Daluwatte, J. H. Miles, S. E. Christ, D. Q. Beversdorf, T. N. Takahashi, and G. Yao, "Atypical pupillary light reflex and heart rate variability in children with autism spectrum disorder," Journal of Autism and Developmental Disorders, vol. 43, no. 8, article no. 1741, pp. 1910-1925, 2013.

[23] P. Kaufman, L. Leonard, and A. Albert, Adler's Physiology of the Eye, Elsevier Health Sciences, 2011.

[24] C. J. K. Ellis, “The pupillary light reflex in normal subjects," British Journal of Ophthalmology, vol. 65, no. 11, pp. 754-759, 1981.

[25] P. H. Heller, F. Perry, D. L. Jewett, and J. D. Levine, "Autonomic components of the human pupillary light reflex," Investigative Ophthalmology \& Visual Science, vol. 31, no. 1, pp. 156-162, 1990.

[26] J. A. Cahill and J. Ross, "Eye on children: Acute work-up for pediatric horner's syndrome. case presentation and review of the literature," Journal of Emergency Medicine, vol. 48, no. 1, pp. 58-62, 2015.

[27] W. F. Maloney, B. R. Younge, and N. J. Moyer, "Evaluation of the causes and accuracy of pharmacologic localization in Horner's syndrome," American Journal of Ophthalmology, vol. 90, no. 3, pp. 394-402, 1980.

[28] H. L. Van der Wiel and J. Van Gijn, “The diagnosis of Horner's syndrome. Use and limitations of the cocaine test," Journal of the Neurological Sciences, vol. 73, no. 3, pp. 311-316, 1986.

[29] R. J. Pandit and R. Taylor, "Mydriasis and glaucoma: Exploding the myth. A systematic review," Diabetic Medicine, vol. 17, no. 10, pp. 693-699, 2000.

[30] S. Beharry and S. Gibbons, "An overview of emerging and new psychoactive substances in the United Kingdom," Forensic Science International, vol. 267, pp. 25-34, 2016.

[31] B. E. Blough, A. Landavazo, A. M. Decker, J. S. Partilla, M. H. Baumann, and R. B. Rothman, "Interaction of psychoactive tryptamines with biogenic amine transporters and serotonin receptor subtypes," Psychopharmacology, vol. 231, no. 21, pp. 4135-4144, 2014.

[32] F. S. Bersani, O. Corazza, P. Simonato et al., "Drops of madness? Recreational misuse of tropicamide collyrium; early warning alerts from Russia and Italy," General Hospital Psychiatry, vol. 35, no. 5, pp. 571-573, 2013.

[33] B. Behnia, M. Heinrichs, W. Bergmann et al., "Differential effects of intranasal oxytocin on sexual experiences and partner interactions in couples," Hormones and Behavior, vol. 65, no. 3, pp. 308-318, 2014.

[34] C. S. Carter, "Oxytocin pathways and the evolution of human behavior," Annual Review of Psychology, vol. 65, pp. 17-39, 2014.

[35] Erowid.com. Erowid Experience Vaults (accessed 07 May 2017). https://erowid.org/experiences/.

[36] The Terence McKenna Page. The Terence McKenna Experience. (accessed 07 May 2017) https://www.facebook.com/groups/terencemckenna/. 
[37] A. E. Neice, M. Behrends, M. P. Bokoch, K. M. Seligman, N. M. Conrad, and M. D. Larson, "Prediction of opioid analgesic efficacy by measurement of pupillary unrest," Anesthesia and Analgesia, vol. 124, no. 3, pp. 915-921, 2017.

[38] T. K. Nuckols, L. Anderson, I. Popescu et al., "Opioid prescribing: a systematic review and critical appraisal of guidelines for chronic pain," Annals of Internal Medicine, vol. 160, no. 1, pp. 38-47, 2014.

[39] K. Rubia, A. A. Alegria, A. I. Cubillo, A. B. Smith, M. J. Brammer, and J. Radua, "Effects of stimulants on brain function in attention-deficit/hyperactivity disorder: A systematic review and meta-analysis," Biological Psychiatry, vol. 76, no. 8, pp. 616628, 2014.

[40] F. Mueller, C. Lenz, M. Steiner et al., "Neuroimaging in moderate MDMA use: a systematic review," Neuroscience and Biobehavioral Reviews, vol. 62, pp. 21-34, 2016.

[41] J. J. López-Ibor, M.-I. López-Ibor, and J. I. Pastrana, “Transcranial magnetic stimulation," Current Opinion in Psychiatry, vol. 21, no. 6, pp. 640-644, 2008.

[42] J. O'Shea and V. Walsh, "Transcranial magnetic stimulation," Current Biology, vol. 17, no. 6, pp. R196-R199, 2007.

[43] B. Phillips, C. Ball, D. Sackett, D. Badenoch, D. Straus, and B. Haynes, "Oxford Centre for Evidence-Based Medicine-Levels of Evidence (March 2009)," (accessed 6 January 2017). http://www .cebm.net/oxford-centre-evidence-based-medicine-levels-evidence-march-2009/.

[44] R. Smith and D. Rennie, "Evidence-based medicine-an oral history," JAMA, vol. 311, no. 4, pp. 365-367, 2014. 


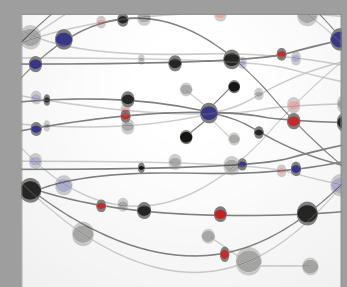

The Scientific World Journal
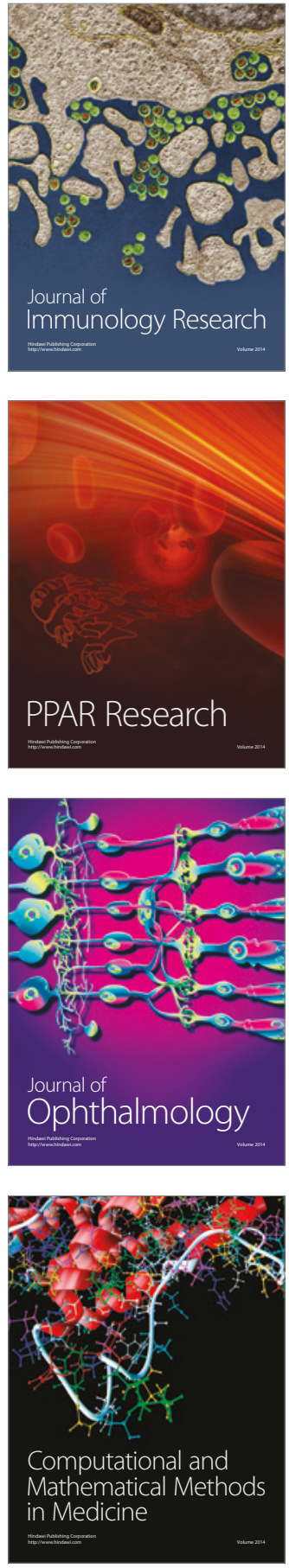

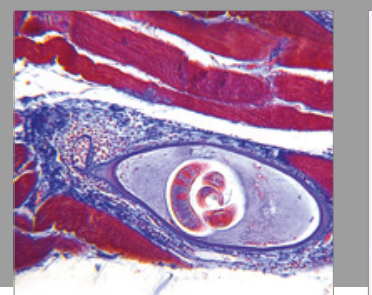

Gastroenterology Research and Practice
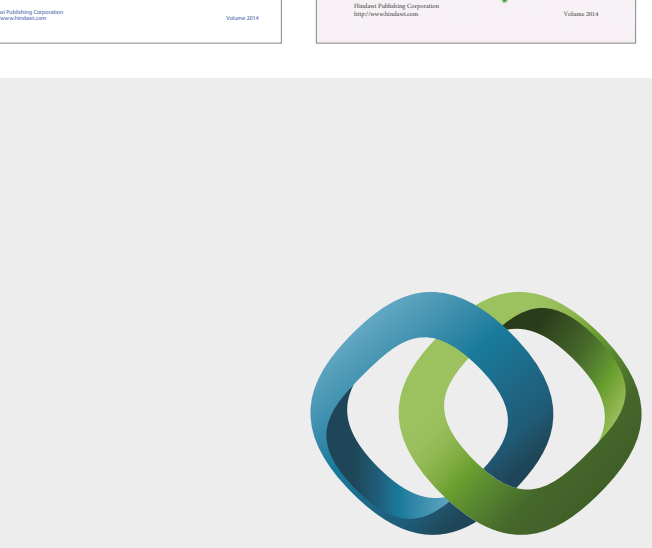

\section{Hindawi}

Submit your manuscripts at

https://www.hindawi.com
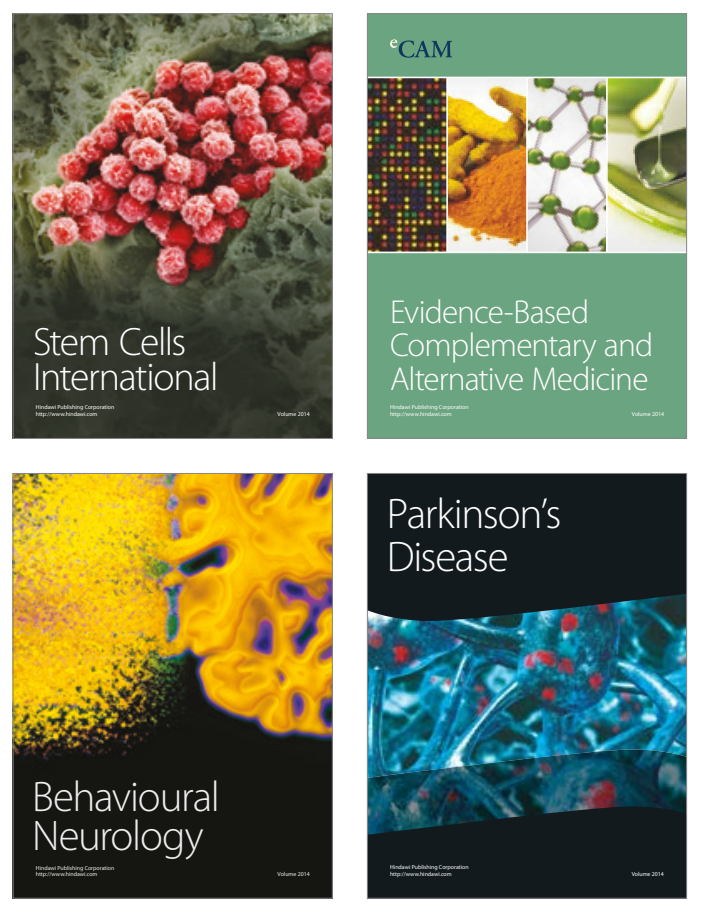
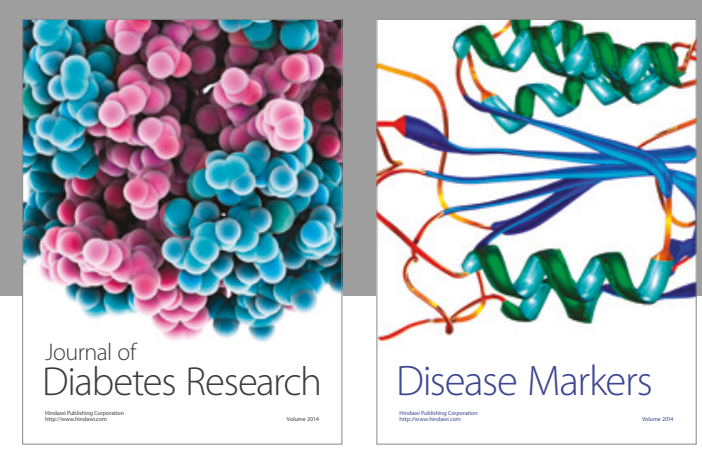

Disease Markers
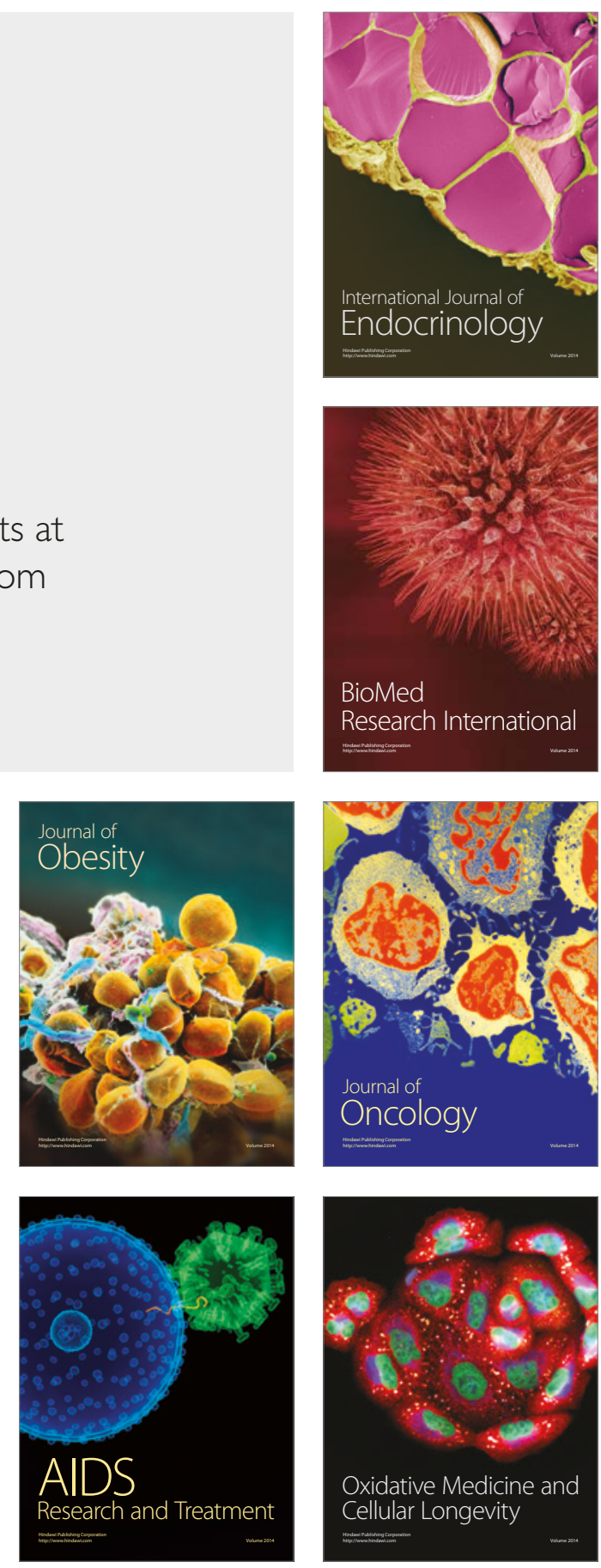